\title{
Electrophysiological abnormalities after Mustard's operation for transposition of the great arteries ${ }^{1}$
}

\author{
Paul C. Gillette, Galal M. El-Said, N. Sivarajan, Charles E. Mullins, Robert L. Williams, \\ and Dan G. McNamara \\ From The Section of Cardiology, Department of Pediatrics, Baylor College of Medicine, and \\ Texas Children's Hospital, Houston, Texas, U.S.A.
}

Because of the frequent occurrence of significant dysrhythmias after Mustard's operation for transposition of the great arteries, electrophysiological studies were performed in 16 patients during postoperative cardiac catheterization. Intracavitary electrical potentials were recorded from the conduction system in all I6 patients. In 9 of these patients, the stability of the atrioventricular conduction system during rapid atrial pacing was tested, as well as the subsequent promptness of pacemaker recovery.

The pacemaker recovery time, corrected for cycle length, was significantly prolonged in 4 of 9 patients studied. After atrial pacing, an escape junctional rhythm preceded the resumption of sinus rhythm in 3 patients. No intra-atrial block was found between the upper and lower intra-atrial electrograms. In 4 patients, an early electrical activity was recorded inside the pulmonary venous (new left) atrium, above the tricuspid valve. This electrical activity occurred during the T-P interval and was unrepresented on the surface electrocardiogram. No significant abnormalities of conduction were observed in the atrioventricular node or the His Purkinje system. Furthermore, rapid atrial pacing caused no abnormal atrioventricular block.

The abnormal pacemaker recovery time and the absence of conduction disturbances in the atria, atrioventricular node, and His-Purkinje system confirm the hypothesis that injury to the sinoatrial node is the main cause of dysrhythmias after Mustard's operation. The pacemaker recovery time may be of prognostic importance in patients after Mustard's operation in identifying those who may develop dysrhythmias.

Patients who have had intra-atrial repair of transposition of the great arteries by the Mustard technique (Mustard, 1964) have often been noted to show atrial dysrhythmias and atrioventricular conduction abnormalities in their scalar electrocardiograms (Khoury et al., 1966; Aberdeen and Carr, 1968; Waldhausen et al., I97I ; El-Said et al., I972; Isaacson et al., 1972; Rodriguez-Fernandez et al., 1972). The main cause of these dysrhythmias was attributed by El-Said et al. (1972) to sinoatrial node injury but Isaacson et al. (I972) proposed surgical interruption of atrial pathways as the cause. Aberdeen and Carr (1968) reported several types of atrioventricular conduction abnormalities and attributed them to damage of the atrioventricular node during the intra-atrial repair.

Received 3 September 1973.

${ }^{1}$ Supported in part by a grant from the National Institutes of Health, United States Public Health Service; and by a grant from the General Clinical Research Branch, National Institutes of Health.
The object of this investigation was to use His bundle electrocardiography and atrial pacing to study electrophysiological phenomena in the sinoatrial node, atria, atrioventricular node, and HisPurkinje system after the intra-atrial repair of transposition of the great arteries.

\section{Subjects and methods}

The following electrophysiological studies were performed during follow-up cardiac catheterizations I month to 6 years after the intra-atrial repair: (I) the atrioventricular and His-Purkinje conduction intervals in 16 patients, (2) the intra-atrial conduction intervals in 10 of these patients, and (3) atrioventricular conduction during rapid atrial pacing, as well as the time and site of pacemaker recovery after rapid atrial pacing, in 9 of these patients.

The ages of the patients at the time the Mustard procedure was performed ranged from I to 24 years. All patients except one had their operations at Texas Children's Hospital. 
TABLE I Dysrhythmias and conduction defects noticed after Mustard's operation in 16 patients studied

AV dissociation by default Junctional rhythm

Atrial flutter and paroxysmal atrial flutter

First-degree AV block

Second-degree AV block

Before operation, all patients had normal sinus rhythm and normal atrioventricular conduction. Decrease of the amplitude of the 'sinus-like' $P$ waves and the appearance of various dysrhythmias (Table I) were observed after operation. During cardiac catheterization, the rhythm in 8 patients varied between junctional and 'sinus-like'. One patient had atrial flutter with $2:$ I block which was converted to junctional rhythm by a mechanically induced premature atrial depolarization. The other I5 patients remained in 'sinus-like' rhythm throughout the study.

All subjects were receiving digitalis and were sedated with pethidine, promethazine, and chlorpromazine according to body weight. A bi- or tripolar electrode catheter was introduced in each patient through a percutaneously placed sheath in the femoral vein. The catheter was manipulated across the mitral valve and withdrawn until a biphasic potential was seen between the atrial and ventricular electrograms in a manner similar to that reported by Scherlag et al. in 1969. In Io of the patients, the catheter was also manipulated to the superior vena cava-systemic venous (new right) atrial junction for recording high atrial potentials. Atrial pacing was performed in 9 patients at the superior vena cava-systemic venous atrial junction using a rate twice the resting rate for 3 minutes. Electrograms from two surface limb leads were recorded before, during, and after sudden cessation of atrial pacing. In 9 patients, a second electrode catheter was introduced through a percutaneously placed sheath in the femoral artery and manipulated across the aortic and tricuspid valves into the pulmonary venous (new left) atrium. A multichannel junction box was used to connect the catheters to an Electronics for Medicine photographic recorder. The electrograms were recorded at paper speeds of both roo and $200 \mathrm{~mm} / \mathrm{sec}$ with one second time lines. By electronic filtration, the frequencies recorded in the intracardiac electrograms were limited to between $40 \mathrm{~Hz}$ and $500 \mathrm{~Hz}$, and in the surface electrocardiogram, to between $0.1 \mathrm{~Hz}$ and $20 \mathrm{~Hz}$.

TABLE 2 Range of corrected pacemaker recovery time and first site of recovery after sudden cessation of rapid atrial pacing in 9 postoperative Mustard patients and in 9 patients with different congenital heart defects who had not had any surgical procedure

\begin{tabular}{lll}
\hline & $\begin{array}{l}\text { After } \\
\text { Mustard's } \\
\text { operation }\end{array}$ & Control \\
\hline Mean age & $5 \cdot 3$ years & 5 years \\
$\begin{array}{l}\text { Corrected pacemaker recovery time } \\
\text { 0-500 msec } \\
600-1200 \text { msec }\end{array}$ & 5 patients & All patients \\
$\begin{array}{l}\text { Site of recovery } \\
\text { Sinoatrial node }\end{array}$ & 4 patients & - \\
Junctional area & 6 patients & All patients \\
\hline
\end{tabular}
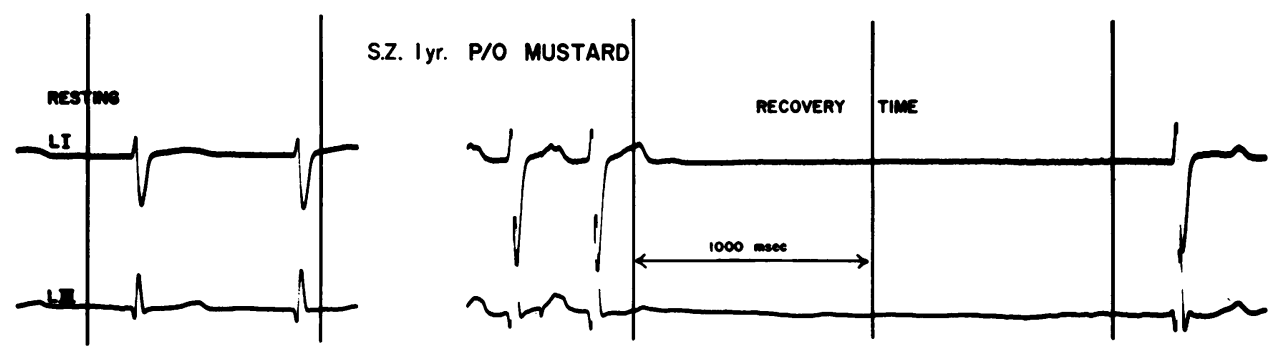

FIG. I Leads I and III before and after sudden cessation of rapid atrial pacing. The corrected pacemaker recovery time, which equals the $R R$ interval after atrial pacing $(2352 \mathrm{msec}$ ) minus the resting $R R$ interval ( $672 \mathrm{msec}$ ), was much prolonged ( $1680 \mathrm{msec}$ ). In this patient, a pacemaker in the junctional area recovered before the sinoatrial node. 
Atrioventricular conduction during rapid atrial pacing and the first pacemaker to recover after sudden cessation of pacing were studied. Because junctional tissues with pacemaker activity recovered earlier than the sinoatrial node in some patients, the $R R$ interval (instead of the PP interval as suggested by Narula, Samet, and Javier (I972)) was used for calculating the corrected pacemaker recovery time. The duration of the resting $R R$ interval before atrial pacing was subtracted from the duration of the interval between the final paced $R$ deflection and the first spontaneous $\mathbf{R}$ wave. The following conduction time intervals were measured: (I) high to low systemic venous atrium, (2) low atrium to His bundle $(\mathrm{AH})$, and (3) His bundle to ventricle (HV). All intervals were measured from the first rapid deflection of one electrical signal to the next. An average value was calculated from 3 to 5 intervals. The corrected pacemaker recovery times were compared statistically (using Student's t test and an IBM computer CCLRES 40) with those obtained from 9 patients of the same age group with various congenital heart lesions who had not had any surgical procedure. Using the same method, the conduction time intervals were also compared to those obtained from 9 patients with transposition of the great arteries and from 71 children with normal conduction studied in our laboratories.

\section{Results}

Corrected pacemaker recovery time (Fig. I and Table 2)

Recovery times $(600,630,650$, and $1200 \mathrm{msec})$ in 4 of the 9 patients who had undergone a Mustard operation exceeded the upper limits found in 9 children with various congenital heart lesions who had not had any surgical procedure, as well as that found in normal adults $(525 \mathrm{msec}$ ) (Narula et al., 1972). All of the patients who had had the Mustard operation and 5 of our control group were receiving digitalis. The junctional area rather than the sinoatrial node was the first site to recover in 2 of the 4 patients with abnormally long recovery times and in I of the 5 with normal recovery. The 4 patients with abnormal recovery times had a greater number of postoperative dysrhythmias and smaller $\mathbf{P}$ waves than the 5 patients with normal recovery times.

\section{Intra-atrial conduction}

In 9 of the Io patients in whom electrograms were recorded from both high and low points in the systemic venous atrium, the earliest electrical
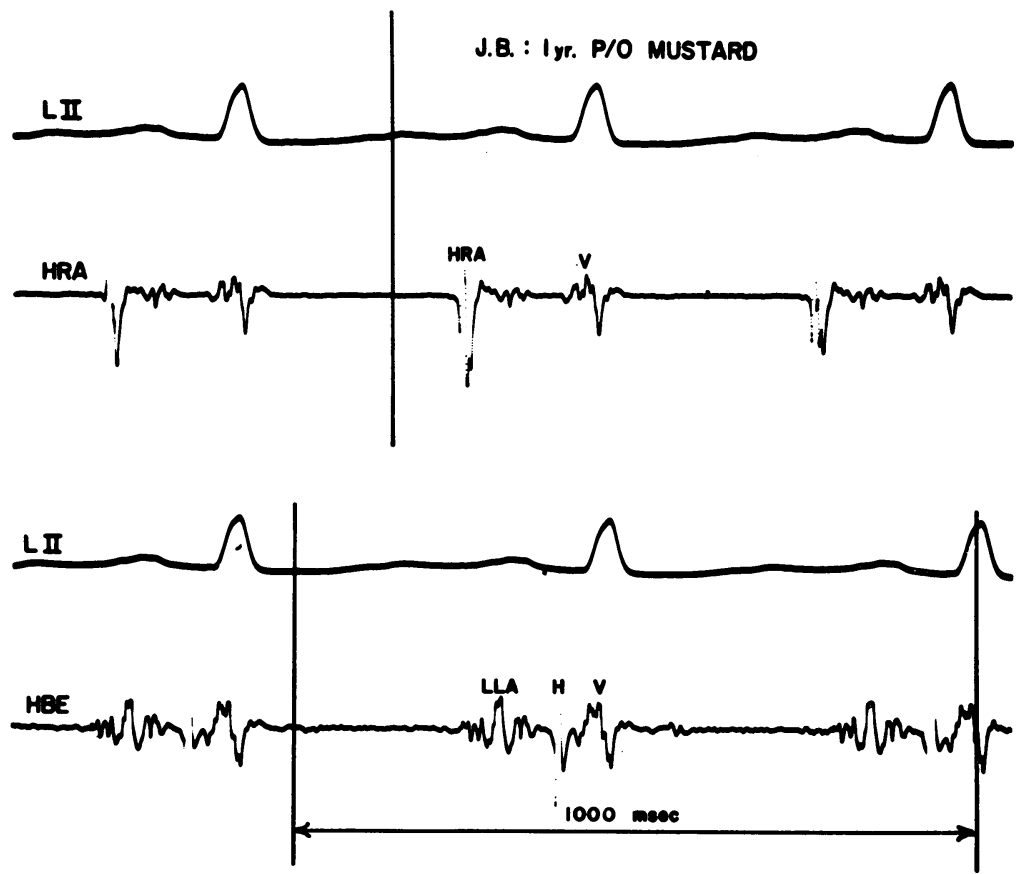

FIG. 2 Lead II and superior vena cava-atrial junction (HRA) and lead II and His bundle electrogram (HBE). There is no abnormal delay between upper (HRA) and lower (LLA) atrial potentials. There is also normal atrioventricular (LLA to $H)$ and His-Purkinje (HV) conduction. 


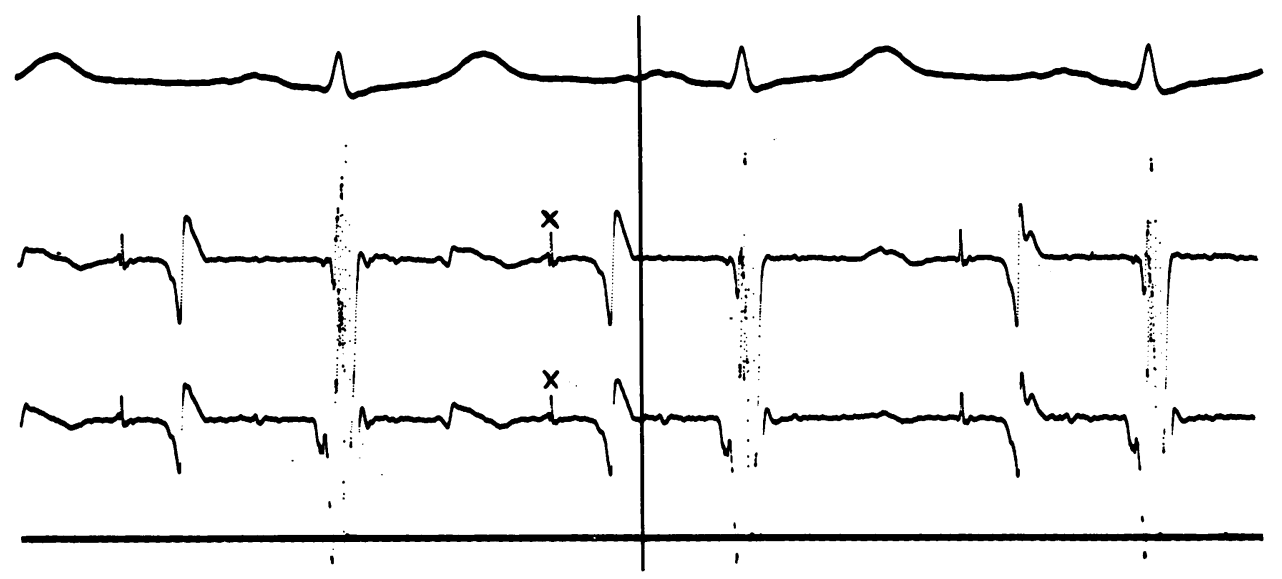

FIG. 3 Lead II and two intra-atrial electrograms obtained by passing the catheter in a retrograde manner across the tricuspid valve into the pulmonary venous (new left) atrium. A biphasic electrical signal (' $X$ ' potential) before the $P$ wave was continuously and repeatedly recorded from the same site.

activity occurred at the junction of the superior vena cava and the right atrium (Fig. 2). No abnormal conduction delay was noticed between the high and low atrium. Also, there was no abnormal delay between the beginning of the surface $P$ wave and the low right atrial electrogram in the 6 patients in whom high and low intra-atrial electrograms were not recorded. In one patient with low atrial rhythm, the earliest electrical activity appeared in the coronary sinus.

In 4 patients with the electrode catheter passed retrogradely through the aorta, across the tricuspid valve and into the pulmonary venous atrium, a previously undescribed ' $X$ ' potential was recorded preceding the surface $P$ wave (Fig. 3). This electrical potential was not represented on the surface electrocardiogram and was a rapid biphasic wave appearing I 25 to $150 \mathrm{msec}$ before the $P$ wave. It could be recorded continuously and repeatedly from the same area in all 4 patients.

\section{Effect of atrial pacing on atrioventricular con- duction}

Increasing the rate of atrial pacing lengthened the PR interval; however, no further atrioventricular block occurred, nor was there any bundle-branch block, with pacing at rates up to 150 to 200 cycles per minute.

\section{Atrioventricular and His-Purkinje conduction} Table 3 shows the mean intervals obtained from the His bundle tracings in the 16 patients after Mustard's operation (Fig. 2), 9 patients with transposition who had not had a Mustard operation, and 7I children with normal conduction. Statistical analysis revealed no differences among the three groups. One patient after operation, as well as one patient with transposition who had not had a Mustard operation, had prolonged HV interval. One patient after Mustard's operation, with low atrial rhythm, had an abnormally short AH interval (29 msec).

TABLE 3 Mean of conduction intervals in postoperative Mustard patients, in children with transposition who had not had Mustard's operation, and those with normal conduction

\begin{tabular}{llllll}
\hline & $\begin{array}{l}\text { No. of } \\
\text { patients }\end{array}$ & $P R$ & P-LoA & LoA-H & $H V$ \\
\hline Mustard & 13 & 134 & 15 & 84 & 43 \\
Transposition of great arteries & 9 & 126 & 17 & 80 & 44 \\
Normal & 71 & 120 & 15 & 78 & 41 \\
\hline
\end{tabular}

LoA $=$ low atrium, $H=$ His potential, $V=$ ventricular potential.

PR interval was obtained from surface electrocardiogram.

There are no statistically significant differences between the intervals. 


\section{Discussion}

Dysrhythmias and conduction defects are significant complications after Mustard's operation for transposition of the great arteries in both the early and late postoperative period. While injury to one or more of the 3 specialized atrial tissues (sinoatrial node, internodal tracts, or atrioventricular node) was considered the cause of these dysrhythmias by some investigators, electrophysiological studies in this investigation confirm our earlier proposal (El-Said et al., 1972) that the main cause of dysrhythmias after Mustard's operation is injury to the sinoatrial node. This injury could result either from direct surgical trauma or indirectly by obliteration of the sinoatrial node artery.

Slow pacemaker recovery time after atrial pacing in several patients was taken as evidence of sinoatrial node dysfunction. The corrected pacemaker recovery time, rather than sinus node recovery time, was used to include subjects in whom the junctional pacemaker recovered before the sinoatrial node. The post-pacing recovery by a junctional pacemaker rather than the sinoatrial node is additional evidence of sinoatrial node damage. Calculation of the corrected sinus node recovery time has been shown to be an effective way to differentiate abnormal from normal sinus node function (Narula et al., 1972;
Rosen, 1972). As previously mentioned, patients with abnormal pacemaker recovery time had a greater number of dysrhythmias and lower amplitude $\mathbf{P}$ waves in the postoperative period than patients with normal pacemaker recovery time. RodriguezFernandez et al. (1972) who observed I: I AV conduction during rapid pacing of the sinoatrial node area, also concluded that damage to the sinoatrial node was the cause of dysrhythmia after the Mustard operation in their patients.

The absence of intra-atrial, atrioventricular, and His-Purkinje conduction abnormalities, even during rapid atrial pacing, provides indirect evidence that sinoatrial node damage is the main cause of dysrhythmias after Mustard's operation. The finding of normal intra-atrial conduction negates the proposal by Isaacson et al. (1972) that disruption of the internodal tracts is responsible for the dysrhythmias after Mustard's operation. While a low atrial pacemaker could mask internodal delay, we found such a condition in only one patient.

A rapid biphasic electrical potential preceding the $P$ wave in the surface electrocardiogram was recorded from the pulmonary venous atrium in 4 patients after Mustard's operation. A similar deflection was not seen in 150 intracardiac recordings in patients with various cardiovascular defects in our laboratories. This previously undescribed ' $\mathrm{X}$ '
Junctional rhythm with

right inferior $\mathrm{p}$ wave axis

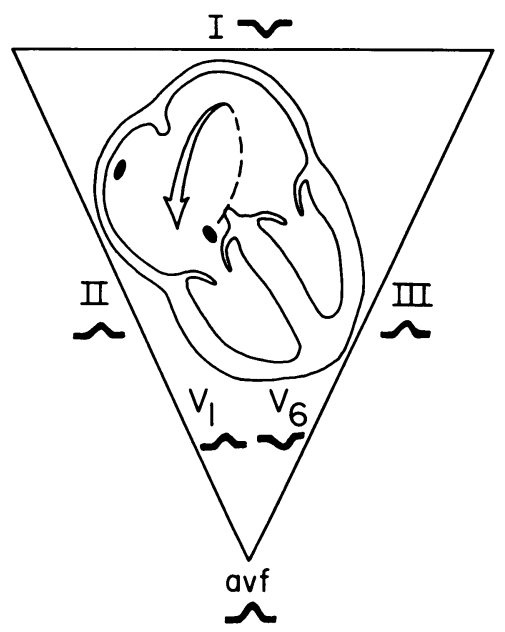

Sinus-like $p$ waves (left inferior $p$ wave axis)

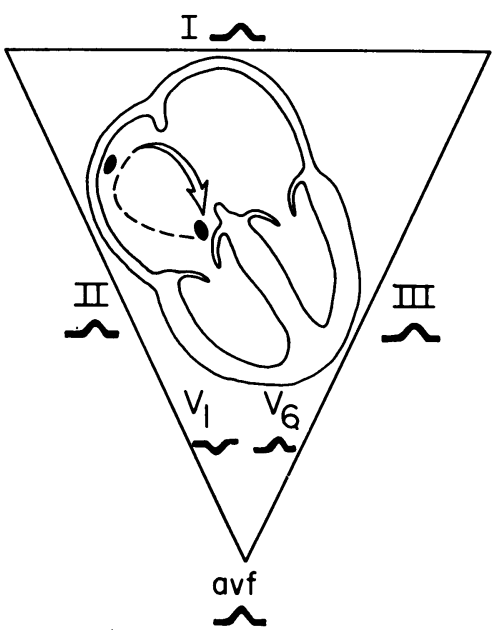

FIG. 4 Diagram depicting the postulated mechanism of junctional rhythm with right inferior $P$ wave axis and 'sinus-like' $P$ waves. The initial silent upward conduction from a junctional pacemaker (dotted line) precedes the second surface-recorded portion (arrow). To explain the direction of the $P$ waves on the surface electrocardiogram, it is postulated that the initial silent conduction in junctional rhythm with right inferior $P$ wave axis is to the left and that the initial silent conduction in 'sinus-like' rhythm is to the right. 
potential may have been recorded because of the unique retrograde positioning of the electrode catheter across the tricuspid valve. Identical biphasic electrical activity could be recorded repeatedly from the same area and could be related more easily to the succeeding $P$ wave than to the preceding $\mathrm{T}$ wave. If this ' $\mathrm{X}$ ' potential is not an artefact, it may represent silent retrograde conduction originating in an atrial or junctional pacemaker. In our earlier report (El-Said et al., 1972), a silent retrograde conduction was proposed to explain the junctional rhythm with right anterior inferior $P$ wave axis and the 'sinus-like' $P$ waves (Fig. 4).

Damage to the sinoatrial node and the existence of abnormal pathways after excision of the interatrial septum and insertion of an intra-atrial baffle to alter the direction of blood flow probably cause the bradytachycardia sick sinus syndrome seen frequently after the Mustard operation.

\section{References}

Aberdeen, E., and Carr, I. (1968). Transposition of the great arteries. In Modern Trends in Cardiac Surgery, Vol. 2, p. 182. Ed. by G. H. Wooler and E. Aberdeen. Butterworth's, London.

El-Said, G., Rosenberg, H. S., Mullins, C. E., Hallman, G. L., Cooley, D. A., and McNamara, D. G. (1972). Dysrhythmias after Mustard's operation for transposition of the great arteries. American fournal of Cardiology, 30, 526.

Isaacson, R., Titus, J. L., Merideth, J., Feldt, R. H., and McGoon, D. C. (1972). Apparent interruption of atrial conduction pathways after surgical repair of transposition of the great arteries. American fournal of Cardiology, 30, 533.

Khoury, G. H., Shaher, R. M., Fowler, R. S., and Keith, J. D. (I966). Preoperative and postoperative electrocardiogram in complete transposition of the great vessels. American Heart fournal, 72, 199.

Mustard, W. T. (I964). Successful two-stage correction of transposition of the great vessels. Surgery, 55, 469.

Narula, O. S., Samet, P., and Javier, R. P. (1972). Significance of the sinus-node recovery time. Circulation, 45, 140.

Rodriguez-Fernandez, H. L., Kelly, D. T., Collado, A., Haller, J. A., Jr., Krovetz, L. J., and Rowe, R. D. (1972). Hemodynamic data and angiographic findings after Mustard repair for complete transposition of the great arteries. Circulation, 46, 799.

Rosen, K. M. (1972). Evaluation of cardiac conduction in the cardiac catheterization laboratory. American fournal of Cardiology, 30, 701 .

Scherlag, B. J., Lau, S. H., Helfant, R. H., Berkowitz, W. D., Stein, E., and Damato, A. N. (1969). Catheter technique for recording His bundle activity in man. Circulation, 39, 13

Waldhausen, J. A., Pierce, W. S., Park, C. D., Rashkind, W. J., and Friedman, S. (1971). Physiological correction of transposition of the great arteries: indications for and results of operation in 32 patients. Circulation, 43, 738.

Requests for reprints to Dr. Paul C. Gillette, Pediatric Cardiology, Texas Children's Hospital, 662I Fannin, Houston, Texas 77025, U.S.A. 